\title{
Virtual Collaboration: The Skills Needed to Collaborate in a Virtual Environment
}

\author{
Shelly R. Roy \\ Fairmont State University, School of Business, Gaston Caperton Center, Clarksburg, USA
}

\begin{abstract}
With support from the scholarly literature, the author of this paper argues that to succeed in virtual collaborative teams (VCTs) individuals need to have relationship building skills, which include the ability to establish trust, familiarity, a positive environment/ context, and embracing diversity, as well as communication skills, which include the use of simple language, ambiguity, and knowledge sharing. The author of this paper further argues that individuals need collaboration skills, which include autonomy/delegation, motivation, adaptability, and innovation, as well as technological skills, which include the use of web collaboration technologies, and virtual worlds/avatars to engage in successful collaboration in virtual environments. In addition, this paper also argues that despite the many advantages of VCTs, many disadvantages to their use still exist.
\end{abstract}

Keywords: Virtual collaboration, virtual collaboration skills, virtual collaboration barriers.

\section{Introduction}

Virtual Collaboration Teams (VCTs), generally defined, are groups of individuals, geographically dispersed, that work together using collaborative technology (e.g. chat rooms, e-mail, instant messaging, video conferencing, etc.) in order to accomplish organizational goals (Brake, 2006; Cottone, Pieti, Schiavinato, Soru, Martinelli, Varotto, \& Mantovani, 2009; Fruchter, Bosch-Sijtsema, \& Ruohomaki, 2010; Suduc, Bizoi, \& Filip, 2009; and Zhang, Tremaine, Egan, Milewski, O'Sullivan, \& Fjermestad, 2009). Many organizations use VCTs because they are inexpensive, independent of time and space, more efficient, more effective, and are better able to share information, than face-to-face teams (Eom, 2009; Muntean, 2009; Suduc, Bizoi, \& Filip, 2009; and Zhang et al., 2009).

Because many organizations use VCTs to accomplish organizational goals, the author of this paper argues that individuals need to have relationship building, communication, collaboration, and technological skills, in order to succeed in VCTs. The author of this paper also argues that because of the unique barriers VCTs create, there is much opposition to their use, and they may not be ideal for all situations. In the subsequent paragraphs, the author supports these arguments with information obtained from scholarly journals.

\section{Relationship Building Skills}

The two principal barriers to VCTsisolation and confusion, are combated through relationship building skills, that create team cohesion, sustainable relationships, encourage trust, team familiarity, and create a shared context among diverse team members (Anonymous, 2010; Brake, 2006; Hastings, 2009; Muntean, 2009; Pyoria, 2009; Robert, Dennis, \& Ahuja, 2008; Shriberg, 2009; and Suduc, Bizoi, \& Filip, 2009). Relationship building skills also help to decrease the intensity of conflicts, encourage the sharing of information, build

Copyright (c) 2012 Shelly R. Roy. This is an open access article distributed under the Creative Commons Attribution License unported 3.0, which permits unrestricted use, distribution, and reproduction in any medium, provided that original work is properly cited. Contact author: Shelly R. Roy E-mail: sroy1@fairmontstate.edu 
a sense of community, and promote clarity throughout the duration of the project (Anonymous, 2010; Brake, 2006; Hastings, 2009; Pyoria, 2009; Robert, Dennis, \& Ahuja, 2008; and Suduc, Bizoi, \& Filip, 2009).

\section{Trust}

Trust is an important element in the collaborative efforts of VCTs, because trust is the foundation for strong team relationships that enable team members to gain mutual respect for one another, to appreciate diversity, share information, and to communicate in an open environment (Archer \& Cameron, 2009; Brake, 2006; Eom, 2009; and Robert, Dennis, \& Ahuja, 2008). Members of VCTs can establish trust by building relationships early on in the project (Brake, 2006), and having at least one initial faceto-face meeting (Shriberg, 2009). However, if a face-to-face meeting is not possible, members of VCTs can post biographical information and pictures online for everyone to see, or team members can meet one another through video conferencing technologies (Brake, 2006; Eom, 2009; and Shriberg, 2009).

\section{Familiarity}

Familiarity is important to the collaboration of VCTs, because after initial trust is established, the members must then begin the process of familiarization by getting to know the cultural background of each member (Eom, 2009), the geographical location of each member, and the knowledge, skills, and abilities each member brings to the collaboration effort (Fedorowicz-Laso-Ballesteros \& PadillaMelendez, 2008). Familiarity also helps to decrease the number of cultural differences that can impede the success of VCTs (Eom, 2009), enhances the ability of VCTs to build team cohesion (Brake, 2006), create an overarching goal that unites the members of VCTs (Shriberg, 2009), and helps to build sustainable relationships within VCTs (Robert, Dennis, \& Ahuja, 2008).

\section{Environment and Context}

To aid the relationship building process, VCTs must create an environment that supports open communication (Zhang et al., 2009), where the members are familiar with one another (Eom, 2009), and where VCTs can engage in active debate (Brake, 2006). In order to create an environment that is conducive to collaboration VCTs must establish a shared context (Cottone, et al., 2009) that unites the diverse members (Hansen, 2009; Innes \& Booher, 2010; and Muntean, 2009). VCTs must also be able to adapt to the shared context as it shifts (David, Chand, Newell, \& ResendeSantos, 2008), and be willing to accept feedback, constructive criticism, and share information, which can be accomplished through the creation of a set of team norms (Archer \& Cameron, 2009; Hansen, 2009; Innes \& Booher, 2010; Sawyer, 2007; and Robinson \& Rose, 2007).

\section{Diversity}

Because VCTs are independent of time and space (Brake, 2006), they are able to have greater diversity than traditional face-toface teams. This is because it is easier to bring together qualified individuals using collaborative technology, than would be to create a team with similar diversity in a face-to-face environment (David, Chand, Newell, \& Resende-Santos, 2008; Fruchter, Bosch-Sijtsema, \& Ruohomaki, 2010; Fruchter \& Ponti, 2010; and Pyoria, 2009). Such added diversity enables VCTs to make better decisions (Anonymous, 2010), by taking advantage of the knowledge, skills, abilities, mindsets, and cultural perspectives of each individual team member (Brake, 2006; David, Chand, Newell, \& Resende-Santos, 2008; Fruchter, Bosch-Sijtsema, \& Ruohomaki, 2010; Fruchter \& Ponti, 2010; Hastings, 2009; Muntean, 2009; Pyoria, 2009; Suduc, Bizoi, \& Filip, 2009; and Zhang, et al., 2009).

\section{Communication Skills}

Communication skills are important to VCTs, because it is through communication 
that the collaborative effort takes place (David, Chand, Newell, \& Resende-Santos, 2008; Fruchter, Bosch-Sijtsema, \& Ruohomaki, 2010; Fruchter \& Ponti, 2010; and Suduc, Bizoi, \& Filip, 2009). In order to ensure that everyone is on the same page in the collaborative effort, VCTs must ensure that their communication is simple and easy to understand, that ambiguity is limited in all messages, and that knowledge is readily shared throughout the entire team (Cottone, et al., 2009; Muntean, 2009; Robert, Dennis \& Ahuja, 2008; and Zhang, et al., 2009).

\section{Simple Language}

In order for VCTs to collaborate in an effective manner, the team must establish a common language, which means that all team members, regardless of their diversity, must use the same definitions for the same words in order to prevent misinterpretations (David, Chand, Newell, \& Resende-Santos, 2008; Fruchter, BoschSijtsema, \& Ruohomaki, 2010; Fruchter \& Ponti, 2010; and Pyoria, 2009). Another way that VCTs can combat misinterpretations during the collaborative effort is to use asynchronous communication (Hastings, 2009), like wikis, forums, and message boards, which generally result in messages that are better thought-out than those presented by synchronous communication (Suduc, Bizoi, \& Filip, 2009).

\section{Ambiguity}

Yet, despite the efforts of using simple language, some ambiguity will always be present in VCTs, because there is a lack of nonverbal communication (Robert, Dennis, \& Ahuja, 2008). To combat this ambiguity, teams can change their preferred method of collaborative technology (Zhang et al., 2009). For example, if a team communicates solely through e-mail and instant messaging, they may try voice-overIP or video conferencing so that everyone can speak to one another in a more natural manner, and so that the team members can see the facial expressions of one another. Other ways to decrease ambiguity are to build strong relationships (Robert, Dennis,
\& Ahuja, 2008), keep the lines of communication open during conflict and message misinterpretation (Brake, 2006; Cottone, et al., 2009; and Shriberg, 2009), establish clearly defined norms/ conventions (Brake, 2006), and to engage in knowledge sharing (Zhang et al., 2009).

\section{Knowledge Sharing}

Knowledge sharing enables VCTs to debate issues, brainstorm, innovate, and share thoughts, experiences, and ideas in a collaborative effort (Fedorowicz, LasoBallesteros, \& Padilla-Melendez, 2008; Pyoria, 2009; Robert, Dennis, \& Ahuja, 2008; and Zhang, et al., 2009). In order for knowledge sharing to occur in VCTs, an environment that is trusting, free of major conflicts, and has strong relationships must be created (Brake, 2006; Eom, 2009; and Hansen, 2009). The environment of VCTs must also be understanding, openly communicative, minimally ambiguous, and supportive of new ideas, in order for knowledge sharing to occur (Brake, 2006; Eom, 2009; Fruchter \& Bosch-Sijtsema, \& Ruohomaki, 2010; Innes \& Booher, 2010; Robert, Dennis, \& Ahuja, 2008; and Zhang et al., 2009).

\section{Collaboration Skills}

Because the purpose of the existence of VCTs is to collaborate, it is only natural that each team member would need a set of collaboration skills in order to succeed in a virtual collaboration team (Brake, 2006; Eom, 2009; Robert, Dennis, \& Ahuja, 2008; and Zhang et al., 2009). These collaboration skills include the ability to work autonomously and delegate tasks (Fruchter, Bosch-Sijtsema, \& Ruohomaki, 2010; and Zhang et al., 2009), the ability to motivate other members of VCTs (Brake, 2006; and Shriberg, 2009), the ability to adapt to changing contexts (Fruchter \& Ponti, 2010), and the ability to innovate (Sawyer, 2007).

\section{Autonomy and Delegation}

VCTs must create team structure by determining the level of autonomy given to 
each team member, and how to delegate tasks equally (Brake, 2006; Eom, 2009; Robert, Dennis, \& Ahuja, 2008; Shriberg, 2009; and Suduc, Bizoi, \& Filip, 2009). Because VCTs are diverse, each team member needs different levels of autonomy (Eom, 2009) and it is the responsibility of the virtual collaborative leader, to delegate tasks in a manner that is equal, but still plays to the knowledge, skills, and abilities of each individual team member (Fruchter, Bosch-Sijtsema, \& Ruohomaki, 2010; Fruchter \& Ponti, 2010; and Muntean, 2009). Autonomy and delegation work in combination with relationship building and communication skills in order to ensure that everyone is working on the same page (Brake, 2006), that each team member is appointed a task that is appropriate for their skill level, and the degree of autonomy they wish to have throughout the collaborative endeavor is maintained (Shriberg, 2009).

\section{Motivation}

Motivation is extremely important to VCTs because it is easy for team members to become frustrated because of technological glitches (Billings, 2009; Cleary, \& MarcusQuinn, 2008; and Nuyens, 2009), language and cultural barriers (Brake, 2006; and Shriberg, 2009), and the increased amount of work that is needed by each team member to participate in VCTs (Muntean, 2009). To keep the spirit of VCTs alive, it is the responsibility of the team leader to provide support to each member of the team, and to empathize with the team members when they voice their frustrations (Brake, 2006; and Shriberg, 2009). However, an optimistic persona (Brake, 2006) and regularly checking-in with each team member on an individual basis are ways that the leaders of VCTs can create sustainable motivation throughout the collaborative project (Brake, 2006).

\section{Adaptability}

VCTs must remain practical with regard to goals set and the deliverables that they promise to the organization (Zhang et al., 2009), because oftentimes the members of VCTs are also members of local face-to- face, and other global VCTs as well (Fruchter \& Ponti, 2010). In addition to other duties, the members of VCTs must also adapt to changes in collaborative technologies (Robert, Dennis, \& Ahuja, 2008), because the average product life cycle in the IT industry is six months (Brake, 2006). Due to the fast-pace of globalized business and the cutthroat competition that many organizations face, VCTs must be willing to adapt and change the focus of the project at a moment's notice (Brake, 2006; and Shriberg, 2009).

\section{Innovation}

The diversity of VCTs is a breeding ground for innovation, and can create a sustainable competitive advantage for an organization (Archer \& Cameron, 2009; Hansen, 2009; Innes \& Booher, 2010; and Sawyer, 2007). In order to encourage innovation, VCTs must create a culture that is conducive to collaboration and uses the knowledge, skills, abilities, and cultural perspectives that each member of the team brings to the collaborative effort (Brake, 2006; Shriberg, 2009; and Suduc, Bizoi, \& Filip, 2009). A common language must be used by the team members to ensure that everyone is on the same page (Robert, Dennis, \& Ahuja, 2008), good relationships must exist in VCTs, and the lines of communication must remain open in order to debate various solutions to the project (Eom, 2009; Fruchter \& Ponti, 2010; Suduc, Bizoi, \& Filip, 2009; Shriberg, 2009; and Sawyer, 2007).

\section{Technological Skills}

In addition to relationship building, communication, and collaboration skills, the members of VCTs also need technological skills in order to succeed in a virtually collaborative team (Brake, 2006; and Shriberg, 2009). This means that the members of VCTs must be proficient in the use of web collaboration technologies, and virtual worlds and avatars (Billings, 2009; Finkel, 2011; Nuyens, 2009; and Owens, Davis, Murphy, Khazanchi, \& Zigurs, 2009). The members of VCTs need technological skills because one of the major problems faced by team members are technical 
glitches, which can result in members of VCTs not participating in the collaborative effort (Brake, 2006; and Shriberg, 2009)

\section{Web Collaboration Technologies}

In order to decrease the amount of technical glitches it is vital to the success of VCTs that all members are using the same technological platform for the collaboration effort (Hastings, 2009). For example, team members using Skype ${ }^{\circledR}$ video conferencing software, cannot communicate with those team members using Go-To-Meeting ${ }^{\circledR} \quad$ voice-over-IP software, and vice versa. However, there are many options for VCTs to choose from where web collaboration technologies are concerned, these options include: chat rooms, e-mail, voice-over-IP, video conferencing, digital whiteboards, forums, message boards, instant messaging, and even the use of virtual worlds/avatars (Brake, 2006; Cottone et al., 2009; Robert, Dennis, \& Ahuja, 2008; and Suduc, Bizoi, \& Filip, 2009). Nevertheless, regardless of the form of web collaboration technology chosen by VCTs, the technology should increase knowledge sharing, manage tasks more efficiently, delegate work in an efficient manner, and decrease misunderstandings and ambiguity among team members (Fedorowicz, LasoBallesteros, \& Padilla-Melendez, 2008).

\section{Virtual Worlds and Avatars}

Virtual worlds, generally defined, are places on the internet that allow the members of VCTs to recreate face-to-face meetings in the virtual realm (Billings, 2009; Finkel, 2011; Nuyens, 2009; and Owens et al., 2009). The members of VCTs participate in these virtual worlds by using avatars, which are digital representations of team members in a virtual world (Billings, 2009; Finkel, 2011; Nuyens, 2009; and Owens et al., 2009). Although virtual worlds and avatars, are relatively new technologies (Owens et al., 2009), they are being used by VCTs in organizations like IBM, NASA, the Massachusetts Institute of Technology (MIT), British Petroleum (BP), and the Harvard Law School (Owens et al., 2009).
What differentiates virtual worlds and avatars from other, more traditional collaborative technologies is the availability of non-verbal communication (Nuyens, 2009; Owens et al., 2009). Because when the members of VCTs use virtual worlds, their avatars can react to comments using gestures, body language, speech, sketch, facial expressions, and touch, which are generally, not available in other collaborative technologies (Nuyens, 2009; Owens, et al., 2009). Because virtual worlds are inexpensive, reduce the cost of travel, and reduce the time to build and maintain physical assets, (Nuyens, 2009) they create a competitive advantage for organizations. VCTs are beginning to exploit the use of avatars and virtual worlds for long distance planning, more effective collaboration, and strategy meetings (Finkel, 2011).

However, because avatars are completely customizable (Billings, 2009; and Finkel, 2011) thirty percent of organizations have put dress codes and behavior codes in place for the avatars of VCTs in order to ensure that avatars are not offensive to the diverse members of VCTs (Finkel, 2011; and Owens et al., 2009).

\section{Opposition}

Despite the advantages of VCTs, which include decreased costs, greater team diversity, enhanced decision-making, more innovation, better communication, and a vast array of other positive aspects (Anonymous, 2010; Brake, 2006; Cottone, et al., 2009; David, Chand, Newell, \& Resende-Santos, 2008; and Shriberg, 2009) there exists in the scholarly literature, much opposition to the use of virtual collaboration teams. The opposition to the use of VCTs includes the superiority of face-to-face communication (Brake, 2006), the increased amount of misinterpretations experienced by VCTs (David, Chand, Newell, \& Resende-Santos, 2008; and Fruchter, Bosch-Sijtsema, \& Ruohomaki, 2010), the social complexity that diversity adds to the collaborative effort (Conklin, 2005), and the barriers faced by VCTs (Brake, 2006; and Shriberg, 2009). 


\section{Face-to-Face Versus Virtual Collaboration}

One of the principal arguments in opposition to the use of VCTs is that collaborative technologies cannot replace face-to-face collaboration (Brake, 2006; Pyoria, 2009; and Shriberg, 2009). This is because the success of VCTs depends on the ability of the team to cross physical and cultural differences (Fedorowicz, LasoBallesteros, \& Padilla-Melendez, 2008), there is more conflict in VCTs than in faceto-face teams (Anonymous, 2010), and it is hard to establish common ground and for the members of VCTs to bond with one another (Anonymous, 2010). Other reasons for the superiority of face-to-face communication include, the inability of some individuals to become comfortable working with VCTs (Cleary \& MarcusQuinn, 2008), the difficulty of knowledge sharing in VCTs (Robert, Dennis, \& Ahuja, 2008), and a lack of non-verbal communication cues (Robert, Dennis, \& Ahuja, 2008). There is also concern over the amount of time that it takes the members of VCTs to type messages on a keyboard when using chat room or instant messaging technologies (Cottone, et al., 2009), and the need for everyone to use the same technological platform (Hastings, 2009). There is also the common problem of technical glitches causing the members of VCTs to cease participating in the collaborative endeavor because of a lack of motivation (Cleary \& Marcus-Quinn, 2008).

\section{Misinterpretations}

Another opposing paradigm to the use of VCTs is that communication in VCTs is more difficult, because of a lack of nonverbal cues, additional information, and concurrent feedback (Robert, Dennis, \& Ahuja, 2008). Communication in VCTs also increases the cognitive load on the participants, because the team members must process the information they receive, and then type their replies when using instant messaging or chat room technologies (Cottone, et al., 2009; and Robert, Dennis, \& Ahuja, 2008). The use of chat room technologies also increases the likelihood that multiple conversations on different topics are occurring simultaneously (Robert, Dennis, \& Ahuja, 2008). Another barrier to VCTs is that members can easily misinterpret e-mail messages, since there is a lack of supporting information in the message (Cottone, et al., 2009; and Robert, Dennis, \& Ahuja, 2008). In addition to message misinterpretations (Robert, Dennis, \& Ahuja, 2008), VCTs must also overcome language barriers (Brake, 2006), cultural barriers (Shriberg, 2009), contextual barriers (Cottone, et al., 2009), and even time-zone barriers (Brake, 2006) in order to ensure that effective communication takes place.

\section{Diversity}

Still another opposing paradigm to the use of VCTs is that the use of diverse teams, adds social complexity (Conklin, 2005) to the collaborative effort, which complicates the dynamics of VCTs. This social complexity causes some scholars (Conklin, 2005; Fruchter, Bosch-Sijtsema, \& Ruohomaki, 2010; Pyoria, 2009; and Robert, Dennis, \& Ahuja, 2008) to argue that homogeneous teams collaborate more effectively than heterogeneous teams. This is because homogeneous teams share a common language, have already established relationships, and have a history together, all of which will help to eliminate conflict in the collaborative effort (Fruchter, Bosch-Sijtsema, \& Ruohomaki, 2010; Pyoria, 2009; and Robert, Dennis, \& Ahuja, 2008).

\section{Virtual Team Barriers}

Yet, the most prevalent opposition to the use of VCTs is the sheer number of barriers that VCTs face, which include physical location, time zones, national, professional, and organizational cultures, and different access to technologies/infrastructure (Fruchter, Bosch-Sijtsema, \& Ruohomaki, 2010). Other barriers faced by VCTs include the lack of non-verbal communication, the behavior of avatars, and the security of virtual worlds (Billings, 2009; Brake, 2006; Finkel, 2011; Nuyens, 2009; and Owens, et al., 2009). Still other barriers that VCTs face include the 
difficulty of information sharing (Robert, Dennis, \& Ahuja, 2008), technological glitches (Cleary \& Marcus-Quinn, 2008), and ambiguity (Cottone, et al., 2009; and David, et al., 2008). As well as diverted attention (Fruchter \& Ponti, 2010), personality differences in the members of VCTs (Eom, 2009), cultural differences, (Brake, 2006; and Conklin, 2005), and a willingness to share information among team members (Eom, 2009).

\section{Conclusion}

In summation, the peruser has seen that the skills needed to collaborate in a virtual environment include relationship building, communication, collaboration, and technological skills. The reader has also seen that despite the many advantages of the use of VCTs, there is still, much opposition to their use, because of the multiple barriers uniquely faced by VCTs, and because many people still prefer faceto-face collaboration to the use of VCTs.

\section{References}

Anonymous. (2010). "Distant Unity: Technologies That Help Improve Collaboration," Strategic Direction, 26(1), 27-31.

Archer, D. \& Cameron, A. (2009). Collaborative Leadership: How to Succeed in an Interconnected World, ButterworthHeinemann, Oxford, England.

Billings, D. M. (2009). "Teaching and Learning in Virtual Worlds," The Journal of Continuing Education in Nursing, 40(11), 489-490.

Brake, T. (2006). "Leading Global Virtual Teams," Industrial and Commercial Training, 38(3), 116-121.

Cleary, Y. \& Marcus-Quinn, A. (2008). "Using a Virtual Learning Environment to Manage Group Projects: A Case Study," International Journal on ELearning, 7(4), 603-621.

Conklin, J. (2005). Wicked Problems \& Social Complexity, In CONKLIN, J., Dialogue mapping: Building shared understanding of wicked problems (pp. 3-41), John Wiley \& Sons Ltd., West Sussex, England.

Cottone, P., Pieti, L., Schiavinato, V., Soru, D., Martinelli, M., Varotto, D., \& Mantovani, G. (2009). "'Solving' Ambiguity in the Virtual Space: Communication Strategies in a Collaborative Virtual Environment," Cognition, Technology, \& Work, 11(2), 151163.

David, G. C., Chand, D., Newell, S. \& Resende-Santos, J. (2008). "Integrated Collaboration across Distributed Sites: The perils of process and the promise of practice," Journal of Information Technology, 23(1), 44-54.

Eom, M. (2009). 'Cross-Cultural virtual Team and Its Key Antecedents to Success,' Journal of Applied Business and Economics, 10(1), 1-14.

Fedorowicz, J., Laso-Ballesteros, I. \& Padilla-Melendez, A. (2008). "Creativity, Innovation, and E-Collaboration," International Journal of E-Collaboration, $4(4), 1-10$.

Finkel, E. (2011). 'Dress for Virtual Success,' ABA Journal, 97(2), 13.

Fruchter, R., Bosch-Sijtsema, P. \& Ruohomaki, V. (2010). "Tension between Perceived Collocation and Actual Geographic Distribution in Project Teams," AI \& Society, 25(2), 183-192.

Fruchter, R. \& Ponti, M. (2010). "Distributing Attention across Multiple Social Worlds," AI \& Society, 25(2), 169181.

Hansen, M. T. (2009). Collaboration: How leaders avoid the traps, create unity, and reap big results, Harvard Business Press, Boston, Massachusetts.

Hastings, R. (2009). 'Collaboration,' Library Technology Reports, 45(4), 7-9.

Innes, J. E. \& Booher, D. E. (2010). Planning With Complexity: An Introduction to Collaborative Rationality for Public Policy, Routledge, New York, New York. 
Muntean, M. I. (2009). "Knowledge Management Approaches in Portal-Based Collaborative Enterprises," Informatica Economica, 13(4), 32-38.

Nuyens, G. (2009). 'Advanced Collaboration Techniques for More Effective Management,' Public Manager, 38(3), 14-18

Owens, D., Davis, A., Murphy, J. D., Khazanchi, D. \& Zigurs, I. (2009). "RealWorld Opportunities for Virtual-World Project Management," IT Professional Magazine, 11(2), 34-41.

Pyoria, P. (2009). "Virtual Collaboration in Knowledge Work: From Vision to Reality," Team Performance Management, 15(7/8), 366-381.

Robert, Jr., L. P., Dennis, A. R. \& Ahuja, M. K. (2008). "Social Capital and Knowledge Integration in Digitally Enabled Teams," Information Systems Research, 19(3), 314334, 392, 394.

Robinson, G. \& Rose, M. (2007). Teams for a new generation: A facilitator's field guide, AuthorHouse, Bloomington, Indiana.

Sawyer, R. K. (2007). Group genius: The creative power of collaboration, Basic Books, New York, New York.

Shriberg, A. (2009). 'Effectively Leading and Managing a Virtual Team,' The Business Review, Cambridge, 12(2), 1-2.

Suduc, A. M., Bizoi, M. \& Filip, F. G. (2009). "Exploring multimedia web conferencing," Informatica Economica, 13(3), 5-17.

Zhang, S., Tremaine, M, Egan, R., Milewski, A., O'Sullivan, P. \& Fjermestad, J. (2009). "Occurrence and Effects of Leader Delegation in Virtual Software Teams," International Journal of E-Collaboration, 5(1), 47-68. 